\title{
ALICE results on heavy-flavour production at the LHC
}

\author{
R. Bailhache on behalf of the ALICE collaboration* \\ IKF Goethe Universitaet Frankfurt \\ E-mail: r.bailhachedikf.uni-frankfurt.de
}

\begin{abstract}
In ultra-relativistic heavy-ion collisions, heavy quarks, i.e. charm and beauty, are of particular interest, since they are produced in the early stage of the reaction and coexist with the surrounding medium. Therefore the measurement of open heavy-flavour production in $\mathrm{Pb}-\mathrm{Pb}$ collisions at the LHC gives access to the mechanisms of heavy-quark transport and energy loss in hot and dense QCD matter. The ALICE apparatus allows us to measure heavy-flavour particles down to low transverse momentum $p_{\mathrm{T}}$, using hadronic and semi-electronic final states at mid-rapidity and semi-muonic final states at forward rapidity. We first present results in pp collisions at center-ofmass energies $\sqrt{s}$ of 2.76 and $7 \mathrm{TeV}$. These measurements provide information on heavy-quark production at LHC energies and constitute the reference for heavy-ion studies. We focus then on the observation of the suppression and azimuthal anisotropy of heavy-flavour production in $\mathrm{Pb}-\mathrm{Pb}$ collisions at $2.76 \mathrm{TeV}$.
\end{abstract}

LHC on the March - IHEP-LHC,

20-22 November 2012

Institute for High Energy Physics, Protvino,Moscow region, Russia

${ }^{*}$ Speaker. 


\section{Introduction}

Ultra-relativistic nuclear collisions allow to study nuclear matter under extreme conditions. A deconfined state of quarks and gluons, the Quark-Gluon Plasma (QGP), is expected to be formed in such collisions. The study of the heavy-quark production is of particular interest since charm and beauty quarks are produced via hard partonic scattering processes at the beginning of the collision and interact strongly with the surrounding medium. Therefore, the measurement of open heavyflavour production and their elliptic flow allows one to test models of in-medium parton energy-loss and probe the level of thermalization of heavy quarks in the medium at low transverse momentum $p_{\mathrm{T}}$. At high $p_{\mathrm{T}}$, the elliptic flow measurement can address the path-length dependence of the heavyquark energy loss. For this purpose the open heavy-flavour production in elementary pp collisions at the same center-of-mass energy has to be as well measured, since it provides the necessary baseline for heavy-ion studies. In addition it tests perturbative Quantum ChromoDynamics (pQCD) calculations in pp collisions.

ALICE (A Large Ion Collider Experiment) collected data during the pp and $\mathrm{Pb}-\mathrm{Pb}$ collisions at center-of-mass energies $\sqrt{s}=7$ and $2.76 \mathrm{TeV}$, and $\sqrt{s_{N N}}=2.76 \mathrm{TeV}$ respectively, delivered by the LHC (Large Hadron Collider) at CERN. A detailed description of the experiment can be found in [1]. At mid-rapidity, open heavy-flavour production is measured in the central barrel via the hadronic decay of D mesons, as well as the semi-electronic decays of heavy-flavour D and B hadrons. At foward rapidity, muons from heavy-flavour hadron decays are reconstructed in the muon spectrometer. The data readout was triggered by a minimum-bias interaction trigger based on the trigger signals from two forward scintillator hodoscopes (VZERO-A and VZERO-C) and two layers of Silicon Pixel Detectors (SPD). The summed amplitudes in the VZERO scintillator tiles were used to determine the centrality of the $\mathrm{Pb}-\mathrm{Pb}$ collisions, employing a model of particle production based on a Glauber description of the nuclear collision geometry. In addition to the minimum-bias trigger, the muon spectrometer provided a single muon trigger at foward rapidity for the heavy-flavour muon analysis. At mid-rapidity, the ElectroMagnetic Calorimeter (EMCal) allowed us to trigger on single electrons for the heavy-flavour elecron analysis.

\section{Results in pp collisions at $\sqrt{s}=7 \mathrm{TeV}$}

In the central barrel of ALICE, the $\mathrm{D}^{0}, \mathrm{D}^{+}, \mathrm{D}^{*+}$ and $\mathrm{D}_{s}^{+}$mesons, and their charge conjugates, are reconstructed from their decays into charged hadrons, $\mathrm{D}^{0} \rightarrow K^{-} \pi^{+}$(with branching ratio, BR of $3.87 \pm 0.05 \%$ ), $\mathrm{D}^{+} \rightarrow K^{-} \pi^{+} \pi^{+}$(BR of $9.13 \pm 0.19 \%$ ), $\mathrm{D}^{*+} \rightarrow \mathrm{D}^{0} \pi^{+}$(BR of $67.7 \pm 0.5 \%$ ) and $\mathrm{D}_{s}^{+} \rightarrow \phi \pi^{+} \rightarrow K^{+} K^{-} \pi^{+}$(BR of $2.32 \pm 0.14 \%$ ) within the rapidity range $|y|<0.5$. Due to their large lifetime (c $\tau=123 \mu \mathrm{m}, 312 \mu \mathrm{m}$ for $\mathrm{D}^{0}$ and $\mathrm{D}^{ \pm}$, respectively), the $\mathrm{D}$ mesons do not decay at the primary vertex. The tracking capabilities of the Inner Tracking System (ITS) and Time Projection Chamber (TPC) are used to reconstruct the displaced secondary vertices. The TPC and Time Of Flight (TOF) detectors provide moreover the possibility to identify $\pi^{ \pm}$and $K^{ \pm}$. The

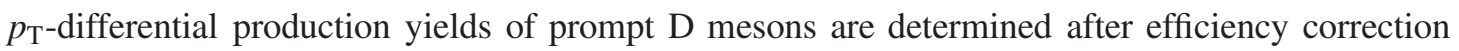
and feed-down correction for $\mathrm{B}$ decays. In pp collisions at $7 \mathrm{TeV}$, the $\mathrm{D}^{0}, \mathrm{D}^{+}$and $\mathrm{D}^{*+}$, and $\mathrm{D}_{s}^{+}$ are measured in the $p_{\mathrm{T}}$ range $1<p_{\mathrm{T}}<16 \mathrm{GeV} / \mathrm{c}, 1<p_{\mathrm{T}}<24 \mathrm{GeV} / \mathrm{c}$ and $2<p_{\mathrm{T}}<12 \mathrm{GeV} / \mathrm{c}$ respectively [2] [3]. The left panel of Fig. 1 shows the measured $p_{\mathrm{T}}$-differential cross section of 

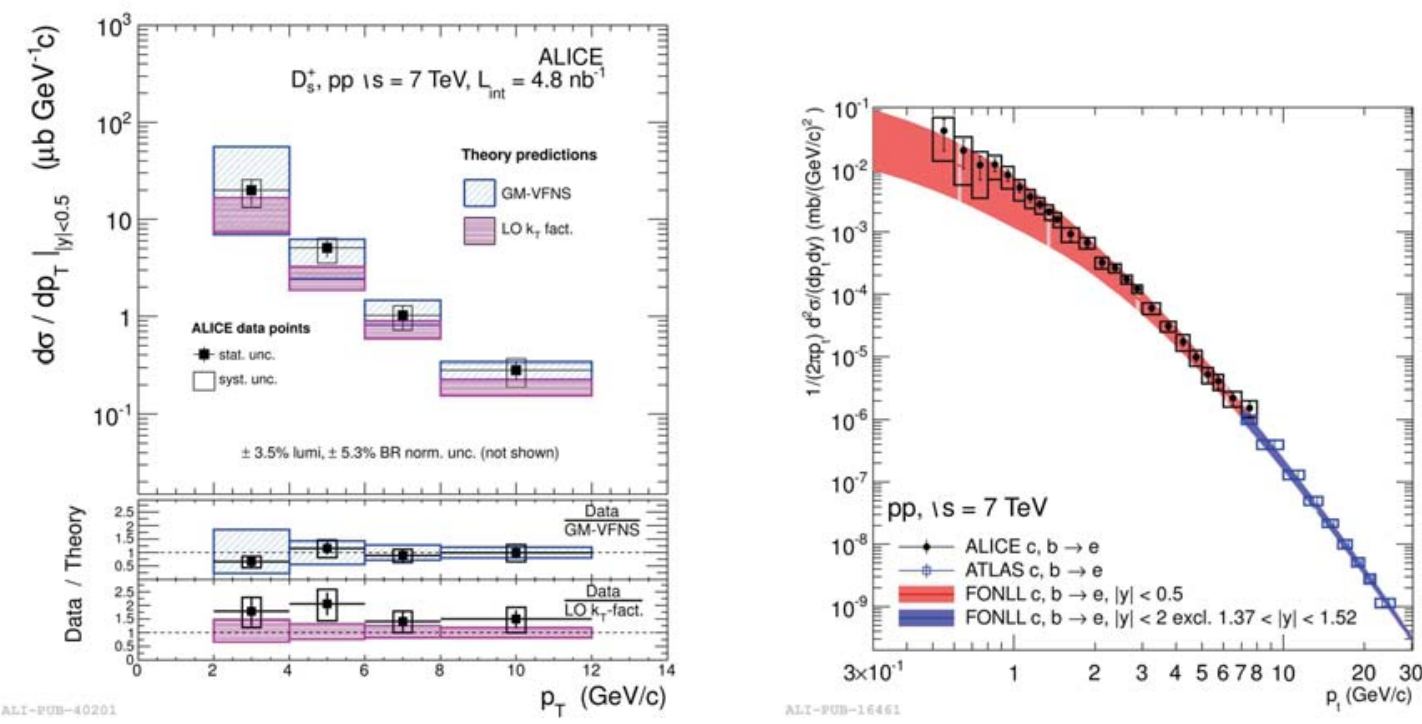

Figure 1: Left: $p_{\mathrm{T}}$-differential cross section of $\mathrm{D}_{s}^{+}$mesons in $\mathrm{pp}$ collisions at $7 \mathrm{TeV}$ compared to $\mathrm{pQCD}$ calculations [3] [17] [16]; Right: $p_{\mathrm{T}}$-differential cross section of electrons from heavy-flavour hadron decays at mid-rapidity $(|y|<0.8)$ in pp collisions at $7 \mathrm{TeV}$, compared to FONLL calculations and ATLAS results at high $p_{\mathrm{T}}[4][5][15]$

$\mathrm{D}_{s}^{+}$mesons compared to different perturbative QCD calculations [17] [16]. The theory describes the data within uncertainties. The pQCD calculations are in reasonable agreement also with the differential cross sections measured for the other D-meson species.

Open heavy-flavour hadrons are measured indirectely via their semi-eletronic decays at midrapidity $(|y|<0.8)$ in the central barrel. Electrons are tracked with the ITS and TPC, and they are identified using different approaches based on the energy loss in the TPC gas, the measured time of flight in TOF, the signal in the Transition Radiation Detector TRD and the measured energy in the EMCal. At low $p_{\mathrm{T}}$, the main contributions to the background due to non heavy-flavour decay electrons come from Dalitz decays of $\pi^{0}$ and from gamma conversions in the detector material. Towards high $p_{\mathrm{T}}$, the signal to background ratio increases. The $\pi^{0}$ spectra measured in ALICE are used as input for the background cocktail, which is subtracted from the measured inclusive electron spectrum to obtain the cross section of electrons from heavy-flavour hadron decays. The right panel of Fig. 1 shows the comparison of the $p_{\mathrm{T}}$-differential cross section of electrons from heavy-flavour hadron decays with FONLL calculations [4]. The theory reproduces well the data. In addition, the ATLAS result extends the ALICE measurement to higher $p_{\mathrm{T}}[5]$.

At forward rapidity $(2.5<y<4.0)$, muons are reconstructed and identified in the muon spectrometer. From the inclusive muon spectrum the muon background component, mainly muons from primary $\pi^{ \pm}$and $K^{ \pm}$decays, must be subtracted. In pp collisions Monte Carlo simulations, using the Phojet and Pythia event generators, are used as input to estimate the muon background at foward rapidity. The right panel of Fig. 2 shows the measured $p_{\mathrm{T}^{-}}$and $y$-differential cross sections of muons from heavy-flavour hadron decays in pp collisions at $\sqrt{s}=2.76 \mathrm{TeV}$, together with FONLL calculations [8] [15]. The ratio between data and FONLL calculations is shown in the 

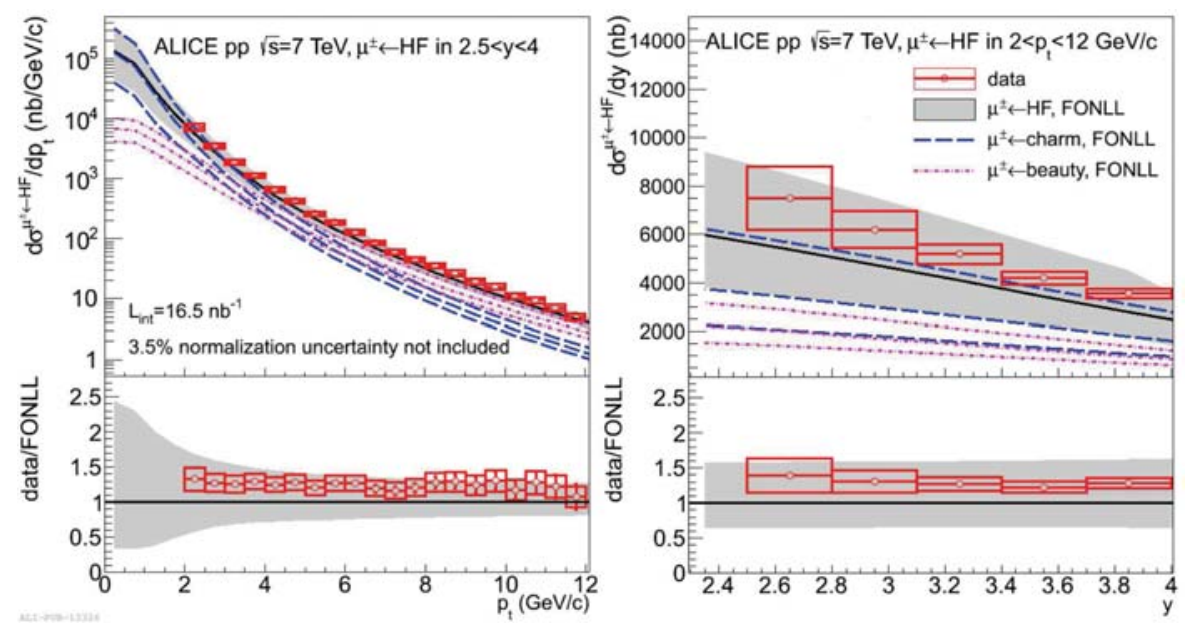

Figure 2: $p_{\mathrm{T}}$-differential cross section of muons from heavy-flavour hadron decays at foward rapidity $(2.5<\mathrm{y}<4)$ in pp collisions at $\sqrt{s}=7 \mathrm{TeV}$, compared to FONLL calculations [8].

bottom panel. The measurements are well reproduced by the model, although at the upper limit of the calculations.

Thanks to the large mass and large lifetime of B hadrons ( $c=500 \mu \mathrm{m}$ ), electrons from Bhadron decays can be separated from electrons from D-hadron decays. Therefore, a significant fraction of the trajectories of electrons from beauty-hadron decays does not point back to the primary collision vertex. Moreover, the electron-hadron correlation in azimuthal angle has a different shape in the near-side for beauty and charm electrons due to decay kinematics. The different approaches to measure electrons from B hadron decays are therefore: an impact parameter analysis with the requirement of a minimum distance of closest approach to the primary vertex for the electron candidate, a B-tagging analysis with the reconstruction of a secondary vertex and an electron-hadron correlation analysis based on the shape of the angular electron-hadron correlation. In pp collisions at $7 \mathrm{TeV}$, electrons from $\mathrm{B}$ hadron decays were measured in the range $1<p_{\mathrm{T}}<$ $13 \mathrm{GeV} / \mathrm{c}$ at mid-rapidity and compared to FONLL calculations [6]. A good agreement with the theory was found.

\section{Results in pp collisions at $\sqrt{s}=2.76 \mathrm{TeV}$}

The same analyses as in pp collisions at $7 \mathrm{TeV}$ were performed in pp collisions at $2.76 \mathrm{TeV}$. At mid-rapidity, the $\mathrm{D}^{0}, \mathrm{D}^{+}$and $\mathrm{D}^{*+}$, and their charge conjugates are measured in pp collisions at $\sqrt{s}=2.76 \mathrm{TeV}$ in the transverse momentum range $1<p_{\mathrm{T}}<12 \mathrm{GeV} / \mathrm{c}$ [7], whereas electrons from heavy-flavour hadron decays are measured from $p_{\mathrm{T}}=2$ to $12 \mathrm{GeV} / \mathrm{c}$. At foward-rapidity, the $p_{\mathrm{T}^{-}}$ differential cross section of muons from heavy-flavour hadron decays is measured in the $p_{\mathrm{T}}$ range $2<p_{\mathrm{T}}<10 \mathrm{GeV} / \mathrm{c}$ [9]. All results are well described within uncertainties by FONLL and GMVFNS calculations [15] [16]. The statistics are nevertheless limited for the D meson and electron analyses and do not allow a comparison with the $\mathrm{Pb}-\mathrm{Pb}$ measurements for every $p_{\mathrm{T}}$ interval. Therefore the reference used for $\mathrm{Pb}-\mathrm{Pb}$ studies is obtained from a pQCD-based (FONLL [15]) energy 


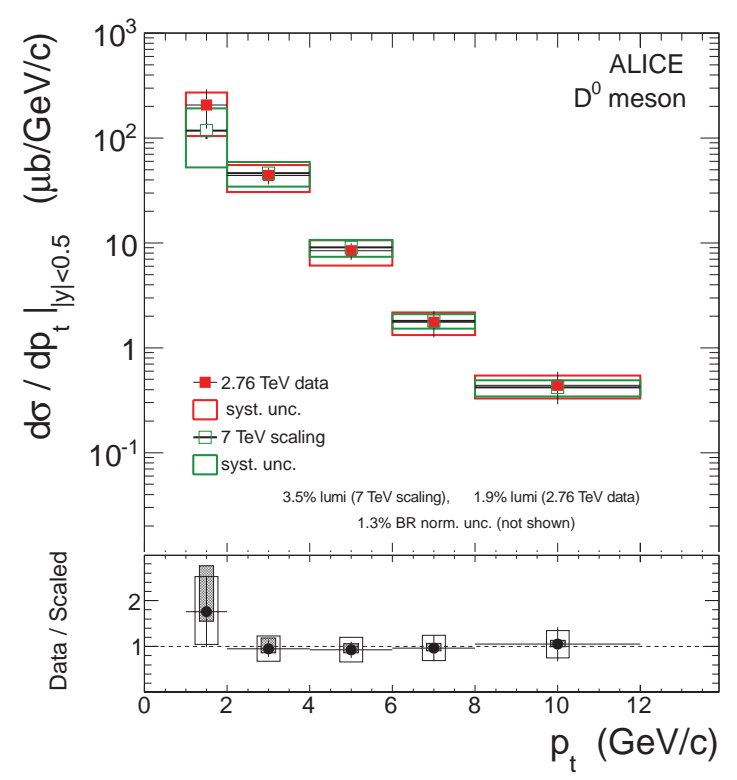

Figure 3: $p_{\mathrm{T}}$-differential cross section for prompt $\mathrm{D}^{0}$ mesons in pp collisions at $\sqrt{s}=2.76 \mathrm{TeV}$ compared to the ALICE measurement at $\sqrt{s}=7 \mathrm{TeV}$ scaled to $2.76 \mathrm{TeV}$ using FONLL (top), together with their ratio (bottom). The filled boxes represent the scaling uncertainties, whereas the empty boxes indicate the measurement systematics [7].

scaling of the $7 \mathrm{TeV} p_{\mathrm{T}}$-differential cross sections to $2.76 \mathrm{TeV}$. Figure. 3 shows the $2.76 \mathrm{TeV}$ measured $p_{\mathrm{T}}$-differential cross section of $\mathrm{D}^{0}$ mesons, together with the $7 \mathrm{TeV}$ results scaled down to $2.76 \mathrm{TeV}$. The agreement is very good. Above $24 \mathrm{GeV} / \mathrm{c}$ for $\mathrm{D}$ mesons and $8 \mathrm{GeV} / \mathrm{c}$ for electrons, no measurement in pp collisions at $7 \mathrm{TeV}$ are available. For the $\mathrm{Pb}-\mathrm{Pb}$ studies, FONLL calculations are then used as reference for heavy-flavour decay electrons, whereas $p_{\mathrm{T}}$ extrapolations using theoretical calculations as baseline for the $p_{\mathrm{T}}$ shape was performed for the different $\mathrm{D}$ meson species. For heavy-flavour decay muons, the $p_{\mathrm{T}}$-differential cross section in pp collisions at $2.76 \mathrm{TeV}$ is directly used as reference for heavy-ion studies.

\section{Results in Pb-Pb collisions at $\sqrt{s_{N N}}=2.76 \mathrm{TeV}$}

The $\mathrm{D}$ meson and heavy-flavour decay lepton production in $\mathrm{Pb}-\mathrm{Pb}$ collisions can be compared to pp collisions at the same energy with the nuclear modification factor $R_{A A}$ :

$$
R_{A A}\left(p_{\mathrm{T}}\right)=\frac{1}{\left\langle T_{A A}\right\rangle} \frac{d N_{A A} / d p_{\mathrm{T}}}{d \sigma_{p p} / d p_{\mathrm{T}}}
$$

where $\left\langle T_{A A}\right\rangle$ is the average nuclear overlap function for the given centrality class which is proportional to the number of binary collisions, $d N_{A A} / d p_{\mathrm{T}}$ is the measured yield for $\mathrm{Pb}-\mathrm{Pb}$ collisions in this centrality class, and $d \sigma_{p p} / d p_{\mathrm{T}}$ is the corresponding cross section in pp collisions. In the absence of any cold and hot matter effects, the nuclear modification factor is 1.0. 

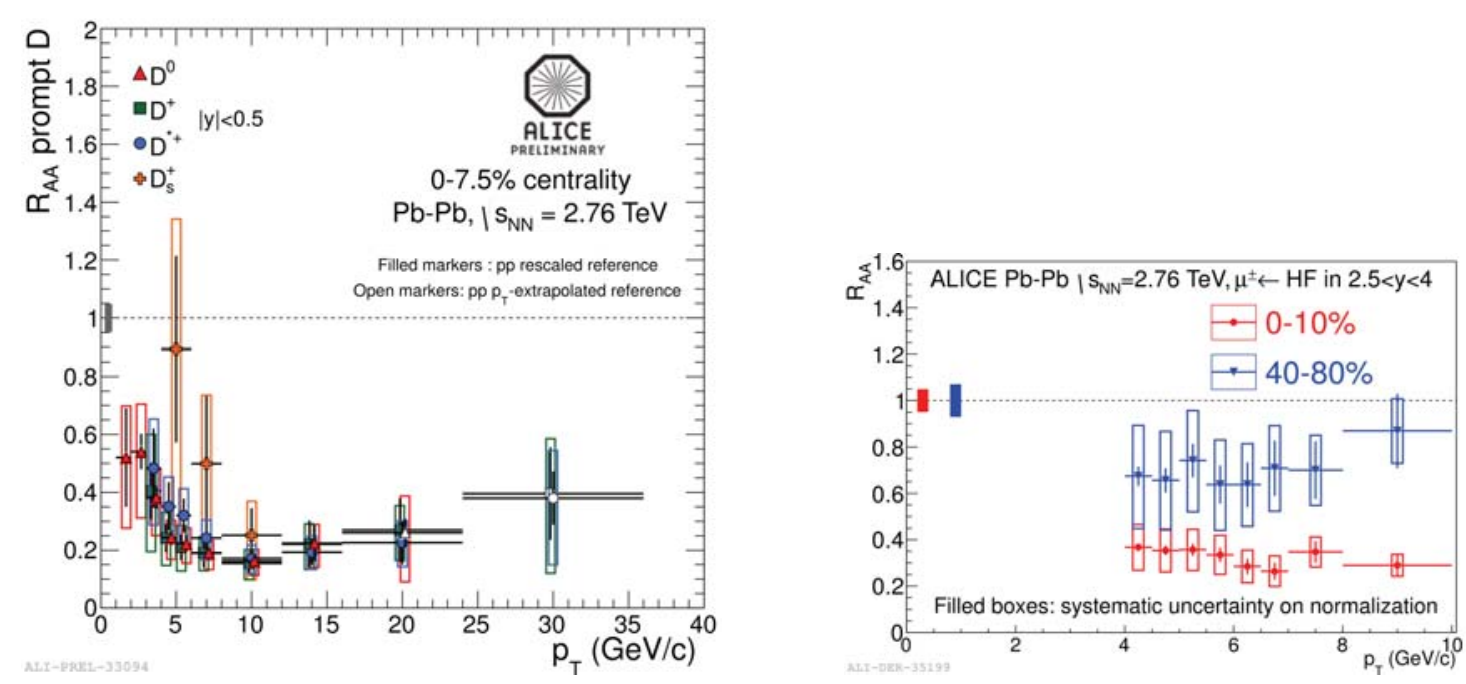

Figure 4: Left: Nuclear modification factor of $D^{0}, D^{+}, D^{+*}$ and $D_{s}^{+}$meson in 0-7.5\% central Pb-Pb collisions at 2.76 TeV. Right: Nuclear modification factor of muons from heavy-flavour hadron decays in $0-10 \%$ and $40-80 \%$ central $\mathrm{Pb}-\mathrm{Pb}$ collisions at $2.76 \mathrm{TeV}[11]$

Prompt D mesons are measured from a $p_{\mathrm{T}}$ of $1 \mathrm{GeV} / \mathrm{c}$ up to a $p_{\mathrm{T}}$ of $36 \mathrm{GeV} / \mathrm{c}$, depending on the D-meson species [10]. A strong suppression compared to pp collisions is observed in the 0$7.5 \%$ and $0-20 \%$ central $\mathrm{Pb}-\mathrm{Pb}$ collisions. The left panel of Fig.4 shows the nuclear modification factors of the different $\mathrm{D}$-meson species in $0-7.5 \%$ central $\mathrm{Pb}-\mathrm{Pb}$ collisions. A similar suppression is observed for all the $\mathrm{D}$ mesons in particular $\mathrm{D}_{s}^{+}$. Some models predicted an enhancement of strange with respect to non-strange $\mathrm{D}$ mesons at intermediate $p_{\mathrm{T}}$ due to recombination of quarks in the medium [12] [13]. [14]. The measurements need better precision for a conclusive statement. A strong suppression compared to pp collisions is also found for the electrons and muons from heavyflavour hadron decays in central $\mathrm{Pb}-\mathrm{Pb}$ collisions at mid-rapidity and foward rapidity, respectively. The right panel of Fig.4 shows the nuclear modification factor of heavy-flavour muons in $0-10 \%$ and $40-80 \% \mathrm{~Pb}-\mathrm{Pb}$ collisions. A smaller suppression is observed in peripheral $\mathrm{Pb}-\mathrm{Pb}$ collisions. The suppression at mid-rapidity of $\mathrm{D}$ mesons and heavy-flavour decay electrons and, at foward rapidity, of heavy-flavour decay muons are similar and compatible taking into account the decay kinematics.

In Fig. 5, the nuclear modification of prompt $\mathrm{D}$ mesons as a function of $p_{\mathrm{T}}$ is compared to the one of charged hadrons [22] and $\pi^{ \pm}[21]$ in central $\mathrm{Pb}-\mathrm{Pb}$ collisions (left panel) and to the one of non-prompt $J / \psi$ from B decays [18] as a function of the mean number of participant in the $\mathrm{Pb}-\mathrm{Pb}$ collisions (right panel). There is an indication for $R_{A A}^{D}>R_{A A}^{\text {charged, } \pi^{ \pm}}$at low $p_{\mathrm{T}}$, whereas the suppression of non-prompt $J / \psi$, although the $p_{\mathrm{T}}$ range is different, is clearly smaller than that of $\mathrm{D}$ mesons in central $\mathrm{Pb}-\mathrm{Pb}$ collisions, taking the decay kinematics into account.

In Fig. 6, the nuclear modification of prompt $\mathrm{D}$ mesons in $0-7.5 \%$ central $\mathrm{Pb}-\mathrm{Pb}$ collisions is compared to the expectation from NLO pQCD [20] with nuclear shadowing [19] and three different models taking into account energy loss at partonic level. The effect of shadowing on the $R_{A A}$ is of the order of $15 \%$ for $p_{\mathrm{T}}>6 \mathrm{GeV} / \mathrm{c}$. This suggests that the strong suppression observed in the data is dominated by final-state effects. The analysis of the $\mathrm{p}-\mathrm{Pb}$ collisions delivered by the LHC in 

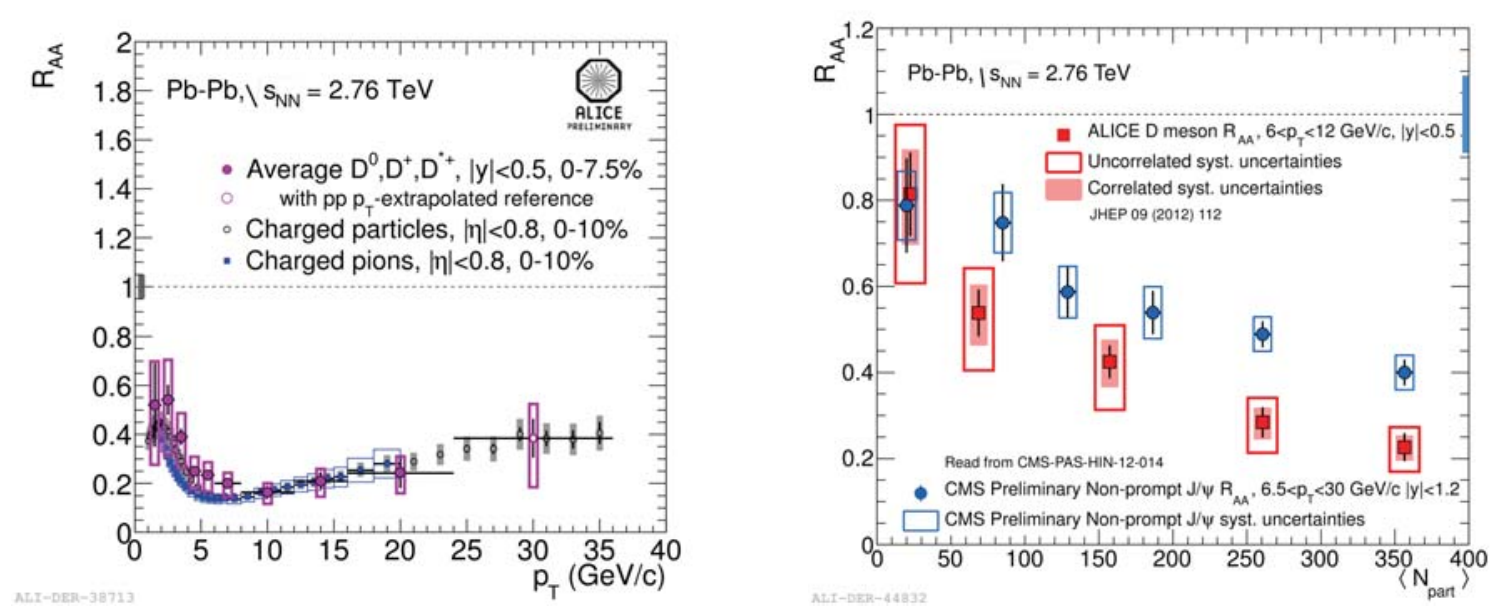

Figure 5: Left: average $R_{A A}$ of $\mathrm{D}$ mesons in $0-7.5 \%$ central $\mathrm{Pb}-\mathrm{Pb}$ collisions at $\sqrt{s_{N N}}=2.76 \mathrm{TeV}$ compared to charged particles and $\pi^{ \pm}$[22] [21] in 0-10\% central collisions; Right: nuclear modification factors of D mesons and non-prompt $J / \psi$ from B decays [18] as function of the average number of participants in $\mathrm{Pb}-\mathrm{Pb}$ collisions at $2.76 \mathrm{TeV}$

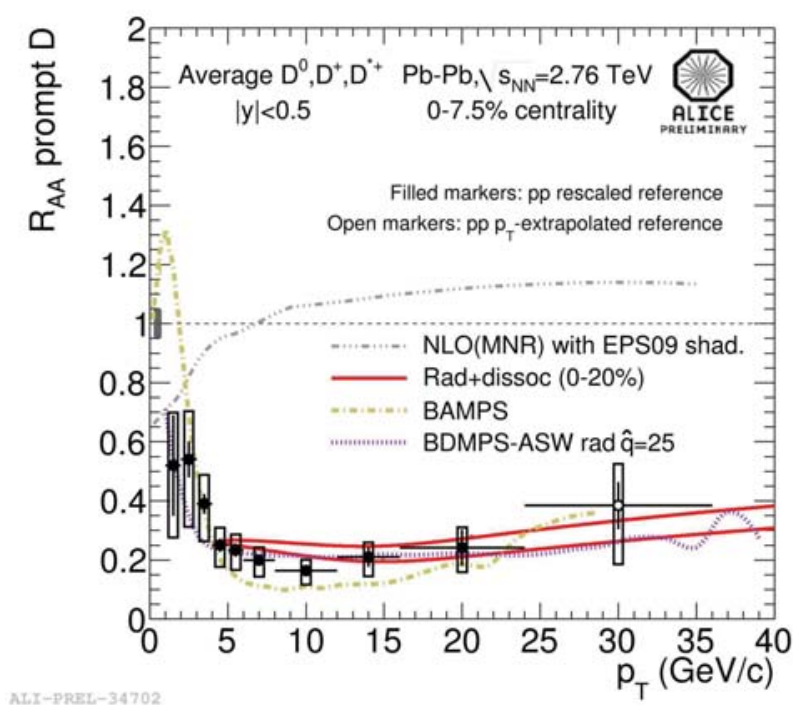

Figure 6: Average $R_{A A}$ of $\mathrm{D}$ mesons in $0-7.5 \%$ central $\mathrm{Pb}-\mathrm{Pb}$ collisions at $\sqrt{s_{N N}}=2.76 \mathrm{TeV}$ compared to model calculations.

January 2013, will allow to measure directly the initial state effects. The models with various parton energy loss mechanisms can describe relatively well the D meson, heavy-flavour leptons, and charged particle nuclear modification factor.

In heavy-ion collisions the initial state spatial anisotropy in non-central collisions is converted into a momentum anisotropy if the in-medium mean free path allows for sufficient rescattering. The resulting azimuthal distribution of the final state particles reflects the initial geometrical anisotropy 
and the transport properties of the medium. The azimuthal dependence of the particle yield can be written in the form of a Fourier series:

$$
E d^{3} N / d^{3} p=\frac{1}{2 \pi} \frac{d^{2} N}{p_{\mathrm{T}} d p_{\mathrm{T}} d y}\left(1+\sum_{n=1}^{\infty} 2 v_{n} \cos \left[n\left(\phi-\Phi_{R}\right)\right]\right)
$$

where $E$ is the energy of the particle, $p$ the momentum, $\phi$ the azimuthal angle, and $\Phi_{R}$ the reaction plane angle. The reaction plane is the plane defined by the beam axis and the impact parameter direction. The second coefficient is the so called elliptic flow $v_{2}$.
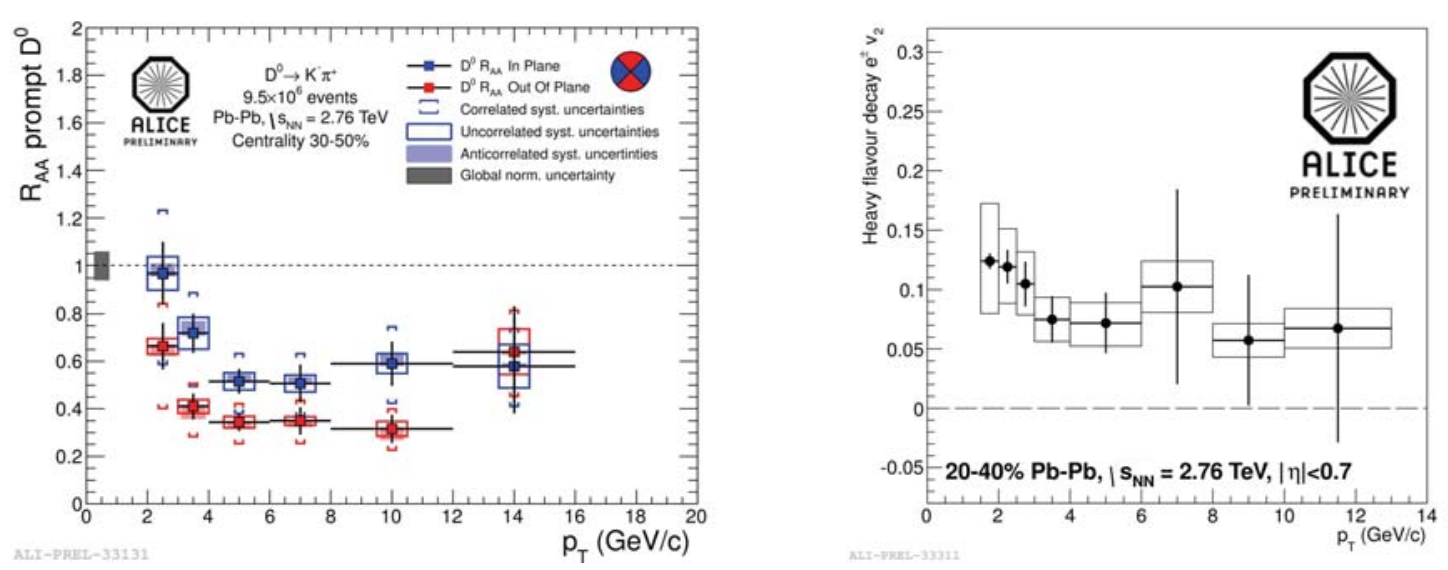

Figure 7: Left: Nuclear modification factor of $\mathrm{D}^{0}$ mesons in- and out-of-plane in $30-50 \%$ central $\mathrm{Pb}-\mathrm{Pb}$ collisions at $\sqrt{s_{N N}}=2.76 \mathrm{TeV}$; Right: Elliptic flow of electrons from heavy-flavour hadron decays in 20-40\% central $\mathrm{Pb}-\mathrm{Pb}$ collisions at $2.76 \mathrm{TeV}$.

The event plane is reconstructed in each event using the TPC or the VZERO detector with a limited resolution. The yield of D mesons or heavy-flavour electrons is then measured with respect to the event plane. The left panel of Fig. 7 shows the $\mathrm{D}^{0}$ nuclear modification factor inand out-of-plane in 30-50\% central Pb-Pb collisions at $\sqrt{s_{N N}}=2.76 \mathrm{TeV}$. For $p_{\mathrm{T}}<12 \mathrm{GeV} / \mathrm{c}$, the observed suppression is larger out-of-plane than in-plane, consistent with the non-zero $v_{2}$ measured for all D-meson species in such collisions. The right panel of Fig. 7 shows the $v_{2}$ of heavy-flavour electrons in 20-40\% central $\mathrm{Pb}-\mathrm{Pb}$ collisions. At low $p_{\mathrm{T}}$, there is an indication for a non-zero $v_{2}$ of electrons from heavy-flavour hadron decays. Fig. 8 shows the nuclear modification factor in 0 $7.5 \%$ central $\mathrm{Pb}-\mathrm{Pb}$ collisions (left panel) and the elliptic flow in $30-50 \%$ central $\mathrm{Pb}-\mathrm{Pb}$ collisions (right panel) compared to different theoretical models. The simultaneous description of $R_{A A}$ and $v_{2}$ is challenging for the models.

\section{Summary and outlook}

We presented the measured $p_{\mathrm{T}}$-differential cross section of D mesons $(|y|<0.5)$, electrons $(|y|<0.8)$ and muons $(2.5<y<4)$ from heavy-flavour hadron decays in pp collisions at $7 \mathrm{TeV}$ and $2.76 \mathrm{TeV}$ with ALICE. The results are reasonably well reproduced by pQCD calculations. In Pb$\mathrm{Pb}$ collisions at $2.76 \mathrm{TeV}$, we showed the measurements of the $\mathrm{D}^{0}, \mathrm{D}^{+}, \mathrm{D}^{*+}$ and $\mathrm{D}_{s}^{+}$mesons 

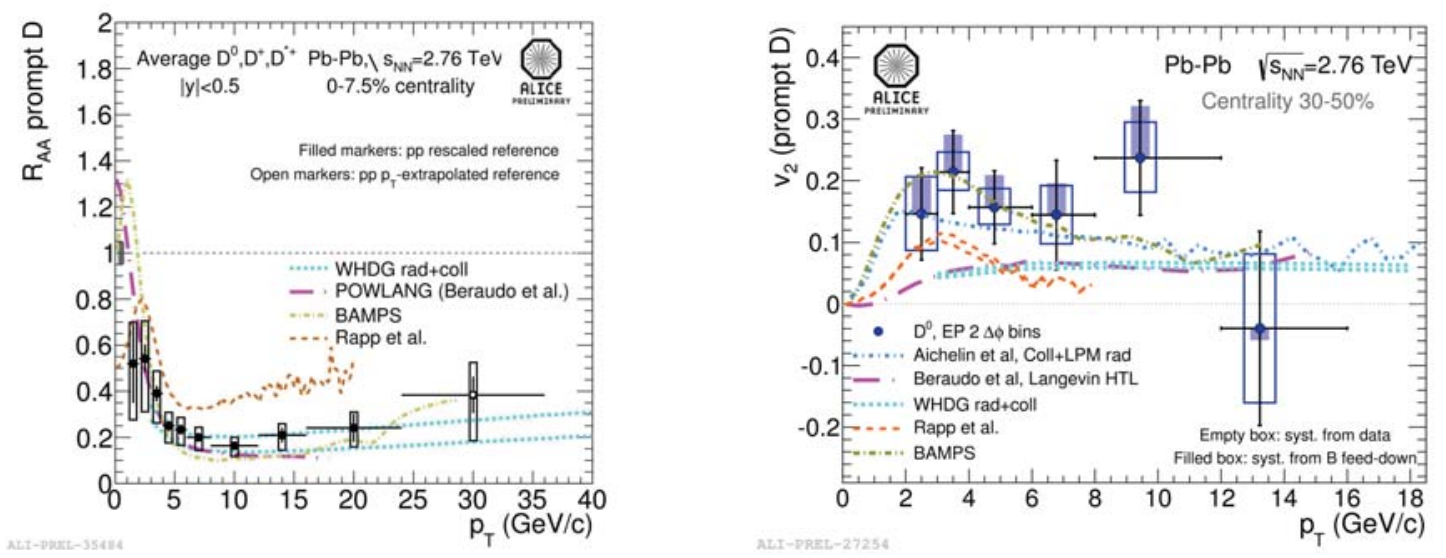

Figure 8: Average $R_{A A}$ of $\mathrm{D}$ mesons in $0-7.5 \%$ central $\mathrm{Pb}-\mathrm{Pb}$ collisions (left) and elliptic flow of $\mathrm{D}$ mesons in $30-50 \%$ central $\mathrm{Pb}-\mathrm{Pb}$ collisions (right) at $\sqrt{s_{N N}}=2.76 \mathrm{TeV}$ compared to different models

and heavy-favour electrons in central collisions at mid-rapidity, as well as heavy-flavour muons in central and mid-peripheral collisions at foward rapidity. A large suppression of open heavyflavour production is observed at high momentum, along with an azimuthal anisotropy with respect to the event plane at low and intermediate $p_{\mathrm{T}}$. Compared to charged hadrons, there is a hint for $R_{A A}^{D}>R_{A A}^{\text {charged, } \pi^{ \pm}}$at low $p_{\mathrm{T}}$. Effects due to shadowing can not explain such a large suppression. At foward rapidity, the suppression of muons from heavy-flavour hadron decays is similar to the one of heavy-flavour electrons at mid-rapidity. Theoretical models with radiative energy loss describe reasonably well at the same time the charm, the heavy-flavour leptons and the charged-hadron suppression. A positive elliptic flow is observed for D mesons and heavy-flavour electrons in semicentral $\mathrm{Pb}-\mathrm{Pb}$ collisions at low $p_{\mathrm{T}}$.

\section{References}

[1] K. Aamodt, JINST, 3:S08002, 2008

[2] B.Abelev et al., JHEP 01 (2012) 128

[3] B.Abelev et al., PL B 718 (2012) 279

[4] B.Abelev et al., PR D 86, (2012) 112007

[5] G.Aad et al., PLB 707 (2012) 438

[6] B.Abelev et al., PL B 721 (2013) 13

[7] B.Abelev et al., JHEP 1207 (2012) 191

[8] B.Abelev et al., PLB 708 (2012) 265

[9] B.Abelev et al., PRL 109 (2012) 112301

[10] B.Abelev et al., JHEP 09 (2012) 112

[11] B.Abelev et al., PRL 109 (2012) 112301

[12] I. Kuznetsova, J.Rafelski, EPJ C51 (2007) 113 
[13] He et al., arXiv:1204.4442

[14] A.Andronic et al., arXiv:0708.1488

[15] M. Cacciari, M. Greco and P. Nason, JHEP 9805007 (1998)

[16] B.A. Kniehl et al., arXiv:1202.0439

[17] R.Maciula et al., arXiv:1208.6126

[18] CMS Collaboration, arXiv:1201.5069 nucl-ex, 2012

[19] K. J. Eskola, H.Paukkunen and C. A. Salgado, JHEP 0904 (2009) 065

[20] M.Mangano, P.Nason and G.Ridolfi, Nucl. Phys. B373 (1992) 295

[21] B.Abelev et al., PRL 109 (2012) 252301

[22] B.Abelev et al., PL B 720 (2013) 52 